\title{
New mosses records (Bryophyta) for Goiás and Tocantins states, Brazil
}

\author{
Denilson Fernandes Peralta ${ }^{1,2}$, Juçara Bordin ${ }^{1}$ and Olga Yano ${ }^{1}$
}

Received: March 30, 2007. Accepted: November 26, 2007

\begin{abstract}
RESUMO - (Ocorrências novas de musgos (Bryophyta) para os Estados de Goiás e Tocantins, Brasil). Após o estudo da coleção do herbário SP, foram encontradas 65 novas ocorrências de musgos para Goiás e Tocantins. Deste total, 44 táxons são primeiras referências (40 - Goiás e quatro - Tocantins) e 23 táxons tiveram a distribuição geográfica ampliada em cada um dos estados (19 - Goiás e quatro - Tocantins). As espécies Ptychostomum pallescens (Schleich. ex Schwägr.) Spence e Philonotis fontana (Hedw.) Brid. são novas citações para o Brasil e apresentam ilustração.
\end{abstract}

Palavras-chave: musgos, Goiás, Tocantins, distribuição geográfica

\begin{abstract}
New mosses records (Bryophyta) for Goiás and Tocantins States, Brazil). Sixty-five moss taxa are new records for Goiás and Tocantins States. Of these, 44 are new records (40 - Goiás; four - Tocantins) and 23 are new sites in each of the states (19 Goiás; four - Tocantins). Ptychostomum pallescens (Schleich. ex Schwägr.) Spence and Philonotis fontana (Hedw.) Brid. are new records for Brazil, and are illustrated.
\end{abstract}

Key words: mosses, Goiás, Tocantins, geographic distribution

\section{Introduction}

Yano \& Costa (2000) carried out the first bryological study for Goiás and Tocantins States, which included sixteen families, nine for Bryophyta and seven for Marchantiophyta. This study reports an important review of bryophyte studies in these states and provides a species checklist to date.

Gradstein \& Costa (2003), studying hepatics, provided descriptions and a list of new records for these states. All of the taxa cited are included in Gradstein et al. (2005), who listed 63 Marchantiophyta species in 16 families. Yano \& Peralta (2007) found 126 Bryophyta species in 26 families for Goiás and Tocantins states and presented descriptions and illustrations.

This work was aimed at researching diversity and knowledge enhancement as regards geographic distribution of bryophyte species for Goiás and Tocantins states.

\section{Material and methods}

After the study of Yano \& Peralta (2007), the authors analyzed bryophyte specimens from the Herbário Científico do Estado Maria Eneyda P. Kauffmann Fidalgo of Instituto de Botânica (SP) and found important new records for Goiás and Tocantins states.
The specimens were identified based on Buck (1998), Crum (1994), Florschütz (1964), Frahm (1991), Ochi (1980;1981), Peralta \& Vital (2006), Sehnem (1970), Sharp et al. (1994), Yano (1979), Yano \& Oliveira-eSilva (1997) and Zander (1993).

The classification system by Buck \& Goffinet (2000) was adopted and geographic distribution in Brazil was based on Yano $(1981 ; 1989 ; 1995 ; 2006)$ and Yano \& Peralta (2007). The species are presented in alphabetical order by family, genus and species.

\section{Results}

Sixty-five moss taxa were found for Goiás and Tocantins states. Forty-four taxa are new records (40Goiás; 4-Tocantins) and the geographic distribution was increased for 23 species (19 - Goiás; four - Tocantins). Two new records are mentioned for Brazil: Ptychostomum pallescens (Schleich. ex Schwägr.) Spence and Philonotis fontana (Hedw.) Brid.

\section{ARCHIDIACEAE}

Archidium julicaule Müll. Hal., Hedwigia 38: 52. 1899.

Illustration: Snider (1975) and Peralta \& Vital (2006).

Specimens examined: BRASIL. Goiás: Formoso $\left(13^{\circ} 12^{\prime} \mathrm{S}, 48^{\circ} 45^{\prime} \mathrm{W}\right)$, on rocky substratum covered with

1 Instituto de Botânica, C. Postal 3005, 01061-970 São Paulo, SP, Brasil

2 Corresponding Author: denilsonfp@yahoo.com.br 
a layer of soil, Fazenda dos Maranhenses, 30/XII/1984, D.M. Vital 12746 (SP 207709).

Brazilian distribution: BA, CE, MG, MS, PB, PE, PI, RN and SE.

\section{BARTAMIACEAE}

Philonotis fontana (Hedw.) Brid., Bryol. Univ. 2: 18. 1827.

Mnium fontanum Hedw., Spec. Musc. Frond.: 195. 1801.

Fig. 1

Specimens examined: BRASIL. Goiás: Formoso $\left(25^{\circ} 23^{\prime} \mathrm{S}, 47^{\circ} 28^{\prime} \mathrm{W}\right)$, sobre rochas recebendo os respingos e a neblina da segunda cachoeira do rio Itiquira, 28/XI/1985, D.M. Vital 13457 (SP 208400).

Reported from Africa, Mexico and North America. First reference for Brazil.

Philonotis uncinata (Schwägr.) Brid., Bryol. Univ. 2: 221. 1827.

Bartramia uncinata Schwägr., Spec. Musc. Frond., Suppl. 2: 60. 1816.

Illustration: Florschütz (1964), Sharp et al. (1994) and Allen (1999).

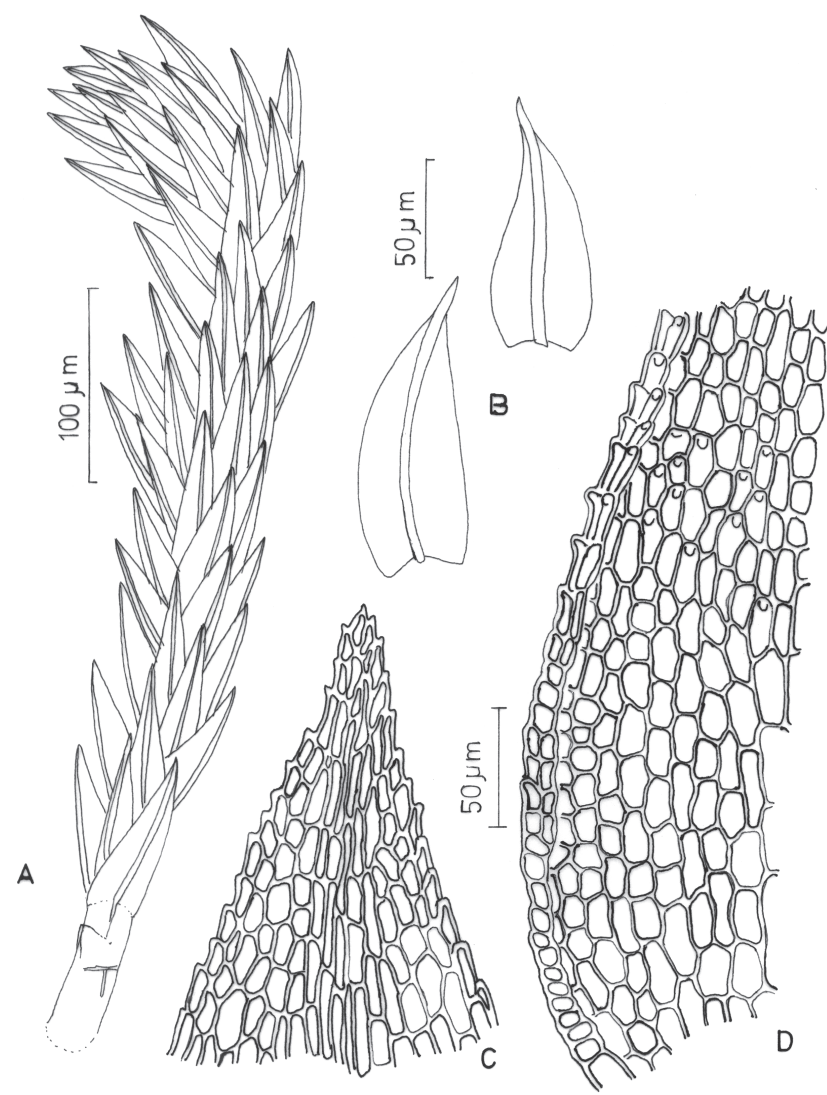

Figure 1. Philonotis fontana (Hedw.) Brid. A. Habit of gametophyte; B. leaves. C. apical cells of leaf. D. marginal basal cells of leaf (SP 208400).
Specimens examined: BRASIL. Goiás: Formoso $\left(13^{\circ} 37^{\prime} \mathrm{S}, 48^{\circ} 49 \mathrm{~W}\right)$, sobre rocha junto à cachoeira da Judith, 7/I/1985, D.M. Vital 12808 (SP 207767).

Brazilian distribution: AC, AM, BA, ES, GO (Aporé; Rio Corrente), MG, MS, MT, PA, PB, PE, PI, PR, RJ, RO, RS, SC, SP, TO (Porto Nacional, Buracão) and DF.

\section{BRACHYTHECIACEAE}

Brachythecium ruderale (Brid.) W.R. Buck, Mem. New York Bot. Gard. 82: 240. 1998.

Hypnum ruderale Brid., Musc. Rec. Suppl. 2: 158. 1812.

Illustration: Buck (1998).

Specimens examined: BRASIL. Goiás: Cocalzinho, nos bordos do Córrego do Barreiro, próximo do $\mathrm{Km} 48$ da BR-70, Fazenda Paraíso, 8/XII/1985, V.R. Estuqui s.n. (SP 208494).

Brazilian distribution: BA, ES, MS, PE, PR, RS and SP. Associated with Cyclodictyon albicans (Hedw.) Kuntze and Lejeunea phyllobola Nees \& Mont.

Squamidium nigricans (Hook.) Broth. in Engler \& Prantl, Natürl. Pflanzenfam. 1(3): 808. 1906. Hypnum nigricans Hook., Syn. Plant. 1: 64. 1822.

Illustration: Sharp et al. (1994) and Buck (1998).

Specimens examined: BRASIL. Goiás: Formoso $\left(25^{\circ} 23^{\prime} \mathrm{S}, 4^{\circ} 28^{\prime} \mathrm{W}\right)$, sobre tronco de árvore, 28/XI/1985, D.M. Vital 13444p.p. (SP 208387).

Brazilian distribution: AP, CE, PE, PR, RJ, RS, SC and SP.

\section{BRUCHIACEAE}

Trematodon longicollis Michx., Flora Bor. Amer. 2: 289. 1803.

Illustration: Sharp et al. (1994) and Churchill \& Linares C. (1995).

Specimens examined: BRASIL. Goiás: Itaberaí, nos bordos do Córrego Rico, 16/XII/1964, D.M. Vital 305 (SP 89251).

Brazilian distribution: AM, ES, MG, PE, PR, RJ, RO, $\mathrm{RS}, \mathrm{SC}, \mathrm{SP}$ and DF.

\section{BRYACEAE}

Bryum paradoxum Schwägr., Spec. Musc. Frond., Suppl. 3(1): 224a. 1827.

Illustration: Ochi (1980, as Bryum paradoxum Schwägr.) and Sharp et al. (1994, as B. beyrichianum).

Specimens examined: BRASIL. Goiás: Formoso $\left(25^{\circ} 23^{\prime} \mathrm{S}, 4^{\circ} 28^{\prime} \mathrm{W}\right)$, solo entre grandes rochas da segunda cachoeira do Rio Itiquira, 28/XI/1985, D.M. Vital 13464 (SP 208406); Cocalzinho, nos bordos do Córrego do Barreiro, próximo do km 48 da BR-70, 
Fazenda Paraíso, 8/XII/1985, V.R. Estuqui s.n. (SP 208496); Goiás (5007’ W, $15^{\circ} 58^{\prime}$ 'S), Serra Dourada, ca. $5 \mathrm{~km}$ de Goiás, camping Santo Antônio, terrestre em barranco úmido, 18/XII/1992, M.R. Silva \& C.E. Rodrigues 559 (SP 322508).

Brazilian distribution: BA, CE, ES, GO (Aporé, Fazenda Cachoeira do Rio Quente), MT, PA, PI, PR, RJ, RR, RS, SC, SP and DF.

Gemmabryum apiculatum (Schwägr.) Spence \& H.P. Ramsay, Phytologia 87: 65. 2005.

Bryum apiculatum Schwägr., Spec. Musc. Frond., Suppl. 1(2): 102. 1816.

Illustration: Sharp et al. (1994, as Bryum apiculatum Schwägr.).

Specimens examined: BRASIL. Goiás: Mineiros, on bared soil, 21/V/1976, D.M. Vital 6376 (SP 135312); Cristalina, Serra dos Cristais, em solo arenoso, ca. $5 \mathrm{~km}$ da cidade, estrada para Paracatu (16 $\left.46^{\circ} \mathrm{S}, 47^{\circ} 37^{\prime} \mathrm{W}\right)$, 4/II/1987, J.R. Pirani et al. 1574 (SP 230700, SPF, K).

Brazilian distribution: AC, AM, BA, MA, MG, PA, PB, RS, SC, SP and DF.

Gemmabryum coronatum (Schwägr.) Spence \& H.P. Ramsay, Phytologia 87: 66. 2005.

Bryum coronatum Schwägr., Spec. Musc. Frond., Suppl. 1(2): 103. 1816.

Illustration: Florschütz (1964) and Sharp et al. (1994) both Bryum coronatum Schwägr..

Specimens examined: BRASIL. Tocantins: Miracema do Norte, parede, 17/II/1974, D.M. Vital 3006 (SP 90853).

Brazilian distribution: AC, AM, BA, GO (Formoso; Rio Paranaíba), MA, MG, MT, PA, PB, PE, PR, RJ, RO, RR, RS, SC, SP, DF and Arquipélago Fernando de Noronha. Associated with Entodontopsis leucostega (Brid.) W.R. Buck \& Ireland.

Gemmabryum exile (Dozy \& Molk.) Spence \& H.P. Ramsay, Phytologia 87: 67. 2005.

Bryum exile Dozy \& Molk., Musci Archip. Ind. Jap.: 3. 1844.

Illustration: Ochi (1980) and Peralta \& Yano (2006), both Brachymenium exile (Dozy \& Molk.) Bosch \& Sande Lac.

Specimens examined: BRASIL. Goiás: Alto Paraíso de Goiás, morro na entrada da cidade, rodovia GO-118 $\left(14^{\circ} 17^{\prime} 48^{\prime \prime} \mathrm{S}, 47^{\circ} 30^{\prime} 54^{\prime \prime} \mathrm{W}\right)$, sobre termiteiro entre arbusto e pedras, $1.250 \mathrm{~m}$ alt., 27/I/1991, O. Yano \& M.P. Marcelli 15160 (SP 231252).

Brazilian distribution: BA, MS, PE, RJ, RR, SP and DF.
Gemmabryum radiculosum (Brid.) Spence \& H.P. Ramsay, Phytologia 87: 68. 2005.

Bryum radiculosum Brid., Spec. Musc. Frond. 3: 18. 1817.

Illustration: Sharp et al. (1994, as Bryum radiculosum Brid.).

Specimens examined: BRASIL. Goiás: Posse, Água Quente, no solo, 8/II/1967, D.M. Vital 1156 (SP 89604); idem, Itaberaí, ca. $18 \mathrm{~km} \mathrm{~S}$ of Itaberaí, on a tree trunk, in a plateau Forest, 28/XII/1974, D.M. Vital 4994 (SP 125759).

Brazilian distribution: MS, MT and SC.

Gemmabryum subapiculatum (Hampe) Spence \& H.P. Ramsay, Phytologia 87: 68. 2005.

Bryum subapiculatum Hampe, Vid. Medd. Naturh. For.

Kjoebenh. ser. 3, 4: 51. 1872.

Illustration: Sharp et al. (1994, as Bryum subapiculatum Hampe).

Specimens examined: BRASIL. Goiás: Paraúna, km 139 along the BR-60, on soil, on bordes of an artificial pond, 20/V/1976, D.M. Vital 6328 (SP 135285); Cristalina, on a swampy area, 17/V/1976, D.M. Vital 6263 (SP 135238).

Brazilian distribution: AM, BA, MT, PA, PR, RJ, RR, RS, SP and DF.

Ptychostomum pallescens (Schleich. ex Schwägr.) Spence, Phytologia 87: 21. 2005.

Bryum pallescens Schleich. ex Schwägr., Spec. Musc. Frond., Suppl. 1(2): 107. 1816.

Fig. 2

Specimens examined: BRASIL. Goiás: Cristalina, Serra dos Cristais, $1050 \mathrm{~m}$ alt., sobre camada de solo em rocha, 4/II/1987, J.R. Pirani et al. 1575 (SP 230701).

Reported from Mexico, New Zealand and South America. First reference for Brazil.

\section{CRYPHAEACEAE}

Schoenobryum concavifolium (Griff.) Gangulee, Mosses Eastern India Adj. Reg. 5: 1209. 1976. Orthotrichum concavifolium Griff., Calcutta J. Nat. Hist. 2: 484.

Illustration: Sharp et al. (1994) and Buck (1998).

Specimens examined: BRASIL. Goiás: Petrolina, sobre tronco de Rubiaceae, região de morros, 6/IX/1979, D.M. Vital 8550 (SP 147855); Alvorada do Norte, on bark tree, 30/V/1978, D.M. Vital 8260p.p. (SP 146956).

Brazilian distribution: AC, AM, BA, ES, GO (Corumbá de Goiás; Goiânia), MG, MS, MT, PE, PR, RJ, RO, RS, SC, SP and DF. Associated with Acrolejeunea emergens (Mitt.) Steph. 


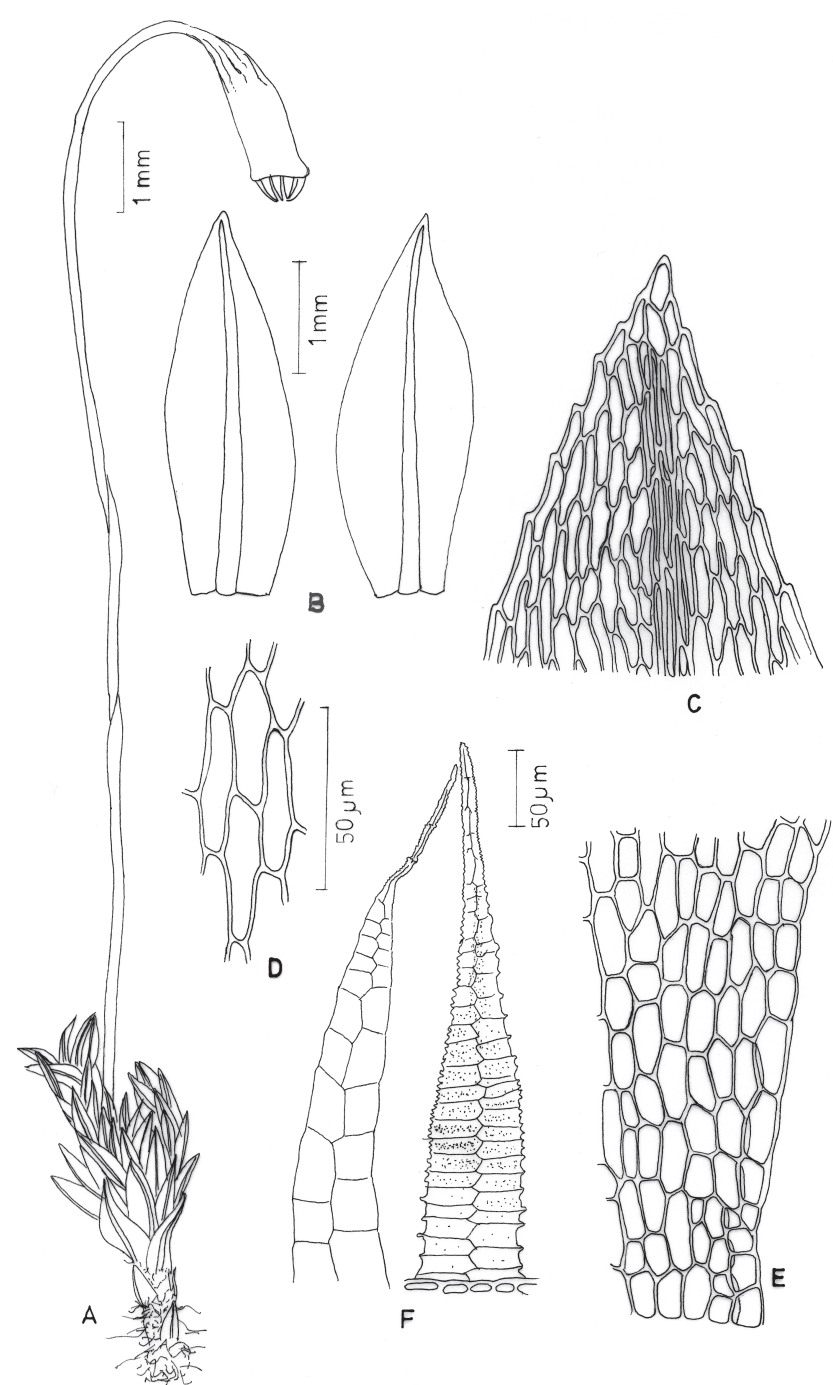

Figure 2. Ptychostomum pallescens (Schleich. ex Schwägr.) Spence. A. Habit of gametophyte. B. leaves. C. apical cells of leaf. D. median cells of leaf. E. marginal basal cells of leaf. F. peristome teeth (SP 274708).

\section{DICRANACEAE}

Anisothecium varium (Hedw.) Mitt., J. Linn. Soc. Bot. 12: 40.1869.

Dicranum varium Hedw., Spec. Musc. Frond.: 133. 1801.

Illustration: Sharp et al. (1994).

Specimens examined: BRASIL. Goiás: Hidrolândia, on walls of a deep cleft, 10/IV/1976, D.M. Vital 6138 (SP 135136); Formoso $\left(13^{\circ} 37^{\prime} \mathrm{S}, 48^{\circ} 45^{\prime} \mathrm{W}\right)$, on banks along a stream at Serra Dourada, 25/XII/1984, D.M. Vital 12680p.p. (SP 207643).

Brazilian distribution: CE, MT and SP.

Campylopus trachyblepharon (Müll. Hal.) Mitt., J. Linn. Soc. Bot. 12: 80. 1869.

Dicranum trachyblepharon Müll. Hal., Syn. Musc. Frond. 1: 389.1848.
Illustration: Frahm (1991).

Specimens examined: BRASIL. Goiás: Cristalina, in partial shade, on a rocky outcrop, 17/V/1976, D.M. Vital 6270 (SP 135243). Tocantins: Babaçulândia, on sandy soil, 16/II/1974, D.M. Vital 2997 (SP 90848).

Brazilian distribution: BA, ES, MG, MT, PR, RJ, RS, SC and SP.

Campylopus uleanus (Müll. Hal.) Broth. in Engler \& Prantl, Natürl. Pflanzenfam. 1(3): 333. 1901. Dicranum uleanum Müll. Hal., Hedwigia 39: 258. 1900.

Illustration: Florschütz (1964) and Frahm (1991). Specimens examined: BRASIL. Goiás: Jataí, on soil in cerrado vegetation, 21/V/1976, D.M. Vital 6359 (SP 135303).

Brazilian distribution: BA, RJ, SC and SP.

Dicranella hilariana (Mont.) Mitt., J. Linn. Soc. Bot. 12: 31. 1869.

Dicranum hilarianum Mont., Ann. Sci. Nat., Bot. ser. 2, 12: 52. 1839.

Illustration: Sharp et al. (1994) and Churchill \& Linares C. (1995).

Specimens examined: BRASIL. Goiás: Goiânia, on humid banks, 20/V/1976, D.M. Vital 6313 (SP 135275); Formoso (13 $37^{\prime}$ 'S, $48^{\circ} 48^{\prime} \mathrm{W}$ ), em barranco úmido junto a nascente, 25/XII/1984, D.M. Vital 12656 (SP 207620).

Brazilian distribution: AC, AM, BA, CE, ES, MG, MS, MT, PA, PB, PE, RJ, RO, RR, SC and SP.

Leucoloma cruegerianum (Müll. Hal.) A. Jaeger, Ber. Thätigh. St. Gallischen Naturwiss. Ges. 1870/71. 412. 1872.

Dicranum cruegerianum Müll. Hal., Syn. Musc. Frond. 2: 588. 1851.

Illustration: Florschütz (1964) and Sharp et al. (1994).

Specimens examined: BRASIL. Goiás: Formoso $\left(13^{\circ} 37^{\prime} \mathrm{S}, 48^{\circ} 45^{\prime} \mathrm{W}\right)$, sobre rocha na encosta da Serra Dourada, 25/XII/1984, D.M. Vital 12675 (SP 207638); idem, on rocks at base of waterfall, Serra Dourada, 1/I/1985, D.M. Vital 12774 (SP 207737).

Brazilian distribution: PE, RJ and SP.

\section{DITRICHACEAE}

Ditrichum subrufescens Broth., Acta Soc. Sci. Fenn. 19(5): 6. 1891.

Illustration: Yano \& Bordin (2006).

Specimens examined: BRASIL. Goiás: Cristalina, ca. $6 \mathrm{~km} \mathrm{SE}$ of town of Cristalina, along the BR-40, on the entrance of a small cave, 17/V/1976, D.M. Vital 6272 (SP 135245). 
Brazilian distribution: MG (Caraça) and RS (São Francisco do Sul).

\section{ENTODONTACEAE}

Entodon beyrichii (Schwägr.) Müll. Hal., Linnaea 18: 708. 1845.

Neckera beyrichii Schwägr., Spec. Musc. Frond., Suppl. sec. 2(2): 162. 1827.

Illustration: Sharp et al. (1994) and Buck (1998).

Specimens examined: BRASIL. Goiás: Itaberaí, sobre pedras nos bordos do riacho, 15/XII/1964, D.M. Vital 301 (SP 89247); Itauçu, Fazenda Córrego Rico, no barranco semipedregoso do afluente do Córrego da Lobeira, 10/XII/1968, M. Vital s.n. (SP 89878).

Brazilian distribution: BA, ES, GO (Moçamedes, Serra Dourada), MG, MS, MT, PA, PE, RJ, RR, RS, SP and DF. Associated with Plagiochila disticha (Lehm. \& Lindenb.) Lindenb.

Entodon macropodus (Hedw.) Müll. Hal., Linnaea 18: 707. 1845.

Neckera macropoda Hedw., Spec. Musc. Frond.: 207. 1801.

Illustration: Sharp et al. (1994) and Buck (1998).

Specimens examined: BRASIL. Goiás: Paraúna, on soil and base of tree trunk, 20/V/1976, D.M. Vital 6332 (SP 135289).

Brazilian distribution: BA, GO (Itauçú), MS and PR.

Erythrodontium longisetum (Hook.) Paris, Ind. Bryol.: 346. 1896.

Neckera longiseta Hook., Musci Exot. 1: 43. 1818.

Illustration: Sharp et al. (1994) and Buck (1998).

Specimens examined: BRASIL. Goiás: Itauçu, Fazenda Córrego Rico, sobre tronco morto, 10/XII/1968, M. Vital s.n. (SP 89877); Paraíso do Norte, on tree trunk, 17/II/1974, D.M. Vital 3013p.p. (SP 90857); Alvorada do Norte, on bark of tree, 30/V/1978, D.M. Vital 8261p.p. (SP 146957); Cristalina, on bark of tree, 31/V/1978, D.M. Vital 8291p.p. (SP 146980).

Brazilian distribution: GO (Goiânia; Campos Belos; Rio Quente), MG, MS, MT, PE, PR, RJ, RS and SP. Associated with Barbula indica (Hook.) Spreng. ex Steud., Frullania gibbosa Nees and Octoblepharum albidum Hedw.

\section{FABRONIACEAE}

Fabronia pusilla Raddi, Atti Acad. Sci Siena 9: 231. 1808.

Illustration: Sharp et al. (1994).

Specimens examined: BRASIL. Goiás: Itaberaí,
Córrego Rico, sobre palmeiras, 10/XII/1968, M. Vital s.n. (SP 89879).

Brazilian distribution: BA and MT.

\section{FISSIDENTACEAE}

Fissidens crispus Mont., Ann. Sci. Nat. Bot. ser. 2, 9: 57. 1838.

Illustration: Sharp et al. (1994).

Specimens examined: BRASIL. Goiás: Formoso

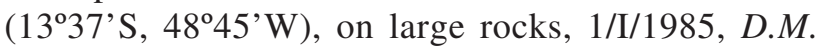
Vital 12761p.p. (SP 207724).

Brazilian distribution: GO (Formoso; Cocalzinho), AM, BA, PR, RS, SC and SP. Associated with Hyophila involuta (Hook.) A. Jaeger.

Fissidens flaccidus Mitt., Trans. Linn. Soc. London 23: 56. 1860.

Illustration: Florschütz (1964) and Sharp et al. (1994), both F. mollis Mitt.

Specimens examined: BRASIL. Goiás: Itaberaí, nos bordos do riacho Córrego Rico, Fazenda Córrego Rico, 19/XII/1964, D.M. Vital 307 (SP 89253).

Brazilian distribution: AC, BA, CE, GO (Caldas Novas; Formoso), MG, MS, MT, PA, PB, PE, PR, RJ, RO, RS, SP and DF.

Fissidens goyazensis Broth., Hedwigia 34: 120. 1895.

Illustration: Sharp et al. (1994).

Specimens examined: BRASIL. Goiás: Itaberaí, ca. $20 \mathrm{~km} S$ da cidade de Itaberaí, sítio do Manoel Vital, no solo, 27/XII/1974, D.M. Vital 4984 (SP 125756).

Brazilian distribution: BA, GO (Formoso; Goiás), PE, SP and Arquipélago Fernando de Noronha.

Fissidens guianensis Mont., Ann. Sci. Nat. Bot. ser. 2, 14: 340. 1840.

Illustration: Florschütz (1964) and Yano \& Oliveirae-Silva (1997).

Specimens examined: BRASIL. Tocantins: Tupirama, Distrito de Guará, sobre cupinzeiro, 16/II/1974, D.M. Vital 3001 (SP 90849); Babaçulândia, sobre cupinzeiro, 16/II/1974, D.M. Vital 2993 (SP 90844).

Brazilian distribution: AC, AM, AP, BA, ES, GO (Alto Paraíso de Goiás), MS, MT, PE, PI, RJ, RO, RR, SP, TO (Porto Nacional) and DF.

Fissidens lagenarius var. muriculatus (Spruce ex Mitt.) Pursell, Bryologist 102(1): 126. 1999. Fissidens muriculatus Spruce ex Mitt., J. Linn. Soc., Bot. 12: 593. 1869.

Illustration: Sharp et al. (1994, as F. diplodus Mitt.). 
Specimens examined: BRASIL. Goiás: Itaberaí, sobre tronco de árvore, mata virgem, 11/XII/1964, D.M. Vital 299 (SP 89245); Formoso, Fazenda Murici, na base da árvore no leito seco da lagoa do Murici, 31/VIII/1979, D.M. Vital 8525 (SP 147835); Montividiu do Norte, cerrado, cupinzeiro na clareira, Fazenda Ipê Amarelo, 22/XII/1998, D.M. Vital s.n. (SP 373079).

Brazilian distribution: AM, ES, PA, PE, RJ, RO, SC and SP.

Fissidens palmatus Hedw., Spec. Musc. Frond.: 145. 1801.

Illustration: Florschütz (1964) and Sharp et al. (1994), both F. reticulosus (Müll. Hal.) Mitt.

Specimens examined: BRASIL. Goiás: Montividiu do Norte, Fazenda Ipê Amarelo, ca. 400 m do Ribeirão Imburuçu, barranco da estrada, mata ciliar, 22/XII/1998, D.M. Vital s.n. (SP 373072); idem, Fazenda Riachão, solo, 24/XII/1998, D.M. Vital s.n. (SP 373084); idem, solo, 21/XII/1998, D.M. Vital s.n. (SP 373064); Formoso, Fazenda Riachão, sobre paredes, 26/XII/1998, D.M. Vital s.n. (SP 373093); idem, solo, 26/XII/1998, D.M. Vital s.n. (SP 373091); idem, no solo, 20/XII/1998, D.M. Vital s.n. (SP 373063).

Brazilian distribution: AC, AM, BA, CE, GO (Goiânia, Bosque dos Buritis, Jardim Botânico), MG, MT, PE, RJ, RO, SP, DF and Arquipélago Fernando de Noronha. Associated with Hyophila involuta (Hook.) A. Jaeger.

Fissidens ramicola Broth., Hedwigia 45: 268. 1906.

Illustration: Sharp et al. (1994).

Specimens examined: BRASIL. Goiás: Formoso, Serra Dourada, paredão rochoso, junto a nascente termal, 2/IX/1979, D.M. Vital 8539p.p. (SP 147845).

Brazilian distribution: AM, BA, CE, ES and GO (Aruanã). Associated with Syrrhopodon prolifer Schwägr.

Fissidens rigidulus Hook. \& Wils. in Hook. f., Flora Nov. Zelandia 2: 61. 1854.

Illustration: Sharp et al. (1994).

Specimens examined: BRASIL. Goiás: Formoso (13 $37^{\circ}$ 'S, $\left.48^{\circ} 45^{\prime} \mathrm{W}\right)$, on rock, 1/I/1985, D.M. Vital 12760p.p. (SP 207723).

Brazilian distribution: MS, MT, RJ and SP. Associated with Philonotis hastata (Duby) Wijk \& Marg.

Fissidens serratus Müll. Hal., Bot. Zeitg 5: 804. 1847.

Illustration: Sharp et al. (1994, as F. papillosus Sande Lac.).

Specimens examined: BRASIL. Goiás: Itaberaí, ca. $18 \mathrm{~km} \mathrm{~S}$ of Itaberaí town, on tree trunk, 28/XII/1974, D.M. Vital 4992p.p. (SP 125757).
Brazilian distribution: BA, GO (Goiânia), MG MT, PE, PI, PR, RJ, RS, SC, SE, SP and DF. Associated with Cheilolejeunea oncophylla (Ångstr.) Grolle \& E. Reiner.

Fissidens submarginatus Bruch in Krauss, Flora 29: 133. 1846.

Illustration: Florschütz (1964) and Sharp et al. (1994), both F. intermedius Müll. Hal..

Specimens examined: BRASIL. Goiás: Montividiu do Norte, Fazenda Ipê Amarelo, cerrado, cupinzeiro, 22/XII/1998, D.M. Vital s.n. (SP 373080); idem, sobre parede, 22/XII/1998, D.M. Vital s.n. (SP 373082); idem, no solo debaixo das mangueiras, 22/XII/1998, D.M. Vital s.n. (SP 373083).

Brazilian distribution: AC, AM, BA, CE, GO (Cristalina; Aruanã), MS, MT, PA, PE, PI, RJ, RO, SC, SP, TO (Porto Nacional; Jalapão), DF and Arquipélago Fernando de Noronha.

Fissidens taylorii Müll. Hal., Syn. Musc. Frond. 1: 65. 1848.

Illustration: Sharp et al. (1994) como F. geheebii Müll. Hal.

Specimens examined: BRASIL. Goiás: Formoso, right margin of Sta. Tereza river $\left(13^{\circ} 12^{\prime} \mathrm{S}, 48^{\circ} 52^{\prime} \mathrm{W}\right)$, on bank along a stream, 29/XII/1984, D.M. Vital 12738 (SP 207701).

Brazilian distribution: SC (Serra Geral and Tubarão).

Fissidens zollingeri Mont., Ann. Sci. Nat. Bot. ser. 3, 4: 114. 1845.

Illustration: Yano et al. (1987) and Sharp et al. (1994).

Specimens examined: BRASIL. Tocantins: Tocantinópolis, na margem do riacho, 15/II/1974, D.M. Vital 2987 (SP 90841).

Brazilian distribution: AC, AM, AP, BA, CE, ES, GO (Formoso; Aruanã; Goiânia; Moçamedes), MA, MG, MS, MT, PA, PB, PE, PI, PR, RJ, RO, RR, SC, SP, TO and Arquipélago Fernando de Noronha.

\section{HELICOPHYLLACEAE}

Helicophyllum torquatum (Hook.) Brid., Bryol. Univ. 2: 771.1827.

Anictangium torquatum Hook., Musci Exot. 1: 41. 1818.

Illustration: Yano (1979), Sharp et al. (1994) and Yano \& Costa (2000).

Specimens examined: BRASIL. Tocantins: Dianópolis, ca. $2 \mathrm{~km}$ ao norte do povoado de Jardim, em fendas de rocha ou pendentes, 12/II/1987, J.R. Pirani et al. 1969 (SP 230708, SPF, K).

Brazilian distribution: AL, AM, BA, CE, ES, GO 
(Alvorada do Norte; Itaberaí; Formosa; Flores de Goiás; Goiânia; Posse; Hidrolândia; Piracanjuba; Paraúna; Caldas Novas; Minaçu; Itumbiara; Moçamedes; Goiás), MG, MS, MT, PA, PB, PE, PI, PR, RJ, SC, SP, TO (Miranorte; Paraíso do Norte) and DF.

\section{HYPNACEAE}

Platygyriella densa (Hook.) W.R. Buck, Brittonia 36: 86. 1984.

Leskea densa Hook. in Kunth, Syn. Plant. 1: 61. 1822.

Illustration: Sharp et al. (1994).

Specimens examined: BRASIL. Goiás: Formoso, sobre rochas junto à fonte termal da Serra Dourada, 2/IX/1979, D.M. Vital 8535p.p., 8538p.p. (SP 147842; SP 147844).

Brazilian distribution: GO (Alto Paraíso, Chapada dos Veadeiros, Rio São Miguel) and DF. Associated with Macromitrium carions Müll. Hal. e Philonotis cernua (Wils.) Griff. \& W.R. Buck.

Taxiphyllum ligulaefolium (E.B. Bartram) W.R. Buck, Trop. Bryol. 2: 42. 1990.

Glossadelphus ligulaefolius E.B. Bartram, Bryologist 49(4): 123. 1946.

Illustration: Sharp et al. (1994, as Glossadelphus ligulaefolius E.B. Bartram) and Buck (1998).

Specimens examined: BRASIL. Goiás: Formoso $\left(13^{\circ} 37^{\prime} \mathrm{S}, 48^{\circ} 45^{\prime} \mathrm{W}\right)$, on banks along a stream, 25/XII/1984, D.M. Vital 12686p.p. (SP 207649); Formosa $\left(25^{\circ} 23^{\prime} \mathrm{S}, 47^{\circ} 28^{\prime} \mathrm{W}\right)$, sobre tronco de árvore vivo, 28/XI/1985, D.M. Vital 13444p.p. (SP 208387).

Brazilian distribution: AM (Manaus). Associated with Meteorium deppei (Müll. Hal.) Mitt., Squamidium nigricans (Hook.) Broth. and Symphyogyna brasiliensis (Nees) Nees \& Mont.

Taxiphyllum taxirameum (Mitt.) M. Fleisch., Musci Buitenzorg 4: 1435. 1923.

Stereodon taxirameus Mitt., J. Proc. Linn. Soc., Bot., Suppl. 2: 105. 1859.

Illustration: Sharp et al. (1994) and Buck (1998). Specimens examined: BRASIL. Goiás: Formoso $\left(13^{\circ} 37^{\prime} \mathrm{S}, 48^{\circ} 45^{\prime} \mathrm{W}\right)$, on rocks at base of a waterfall, Serra Dourada, 1/I/1985, D.M. Vital 12770 (SP 207733).

Brazilian distribution: BA, MS, MT, RJ, SP and TO (Jalapão).

Vesicularia vesicularis (Schwägr.) Broth. in Engler \& Prantl, Natürl. Pflanzenfam. 1(3): 1094. 1908. Hypnum vesiculare Schwägr., Spec. Musc. Frond., Suppl. 2(2): 167. 1827.

Illustration: Sharp et al. (1994) and Buck (1998).
Specimens examined: BRASIL. Goiás: Mineiros, on borders of a stream in a gallery Forest, 21/V/1976, D.M. Vital 6365, 6367 (SP 135306; SP 135308).

Brazilian distribution: AC, AM, ES, GO (Aporé; Quirinópolis; Itajá; Rio Quente), MG, MS, MT, PA, PE, PI, RJ, RO, RR, RS, SC and SP.

Vesicularia vesicularis var. portoricensis (Brid.) W.R. Buck, Brittonia 36: 181. 1984.

Leskea rutilans var. portoricensis Brid., Bryol. Univ. 2: 332. 1827.

Illustration: Buck (1998).

Specimens examined: BRASIL. Goiás: Formoso, Fazenda Murici, sobre tronco podre, no solo da mata de galeria, 30/VIII/1979, D.M. Vital 8512 (SP 147827).

Brazilian distribution: BA, MS and MT.

\section{LEUCODONTACEAE}

Pterogoniadelphus assimilis (Müll. Hal.) Ochyra \& Ziglstra, Táxon 53(3): 810. 2004.

Neckera assimilis Müll. Hal., Syn. Musc. Frond. 2: 92. 1850.

Illustration: Sehnem (1970) and Sharp et al. (1994, as Forsstroemia producta (Hornsch.) Paris).

Specimens examined: BRASIL. Goiás: Paraúna, on rock, in a clump of tree, 20/V/1976, D.M. Vital 6321 (SP 135284); Goiás $\left(50^{\circ} 08^{\prime} \mathrm{W}, 15^{\circ} 53^{\prime} \mathrm{S}\right)$, camping Sto. Antonio, ca. de $7 \mathrm{~km}$ da rod. GO-70, local úmido e sombreado, 18/XII/1993, C.E. Rodrigues Jr. \& M.R. Silva 309 (SP 322226, SJRP).

Brazilian distribution: MG, PR, RJ, RS, SC and SP.

\section{METEORIACEAE}

Meteorium deppei (Hornsch. ex Müll. Hal.) Mitt., J. Linn. Soc. Bot. 12: 441. 1869.

Neckera deppei Hornsch. ex Müll. Hal., Syn. Musc. Frond. 2: 136. 1850.

Illustration: Sharp et al. (1994) and Buck (1998).

Specimens examined: BRASIL. Goiás: Formoso $\left(25^{\circ} 23^{\prime} \mathrm{S}, 4^{\circ} 28^{\prime} \mathrm{W}\right)$, sobre tronco de árvore, 28/XI/1985, D.M. Vital 13444p.p. (SP 208387).

Brazilian distribution: BA, CE, ES, MG, MS, PE, PR, RJ, RS, SP and DF. Associated with Taxiphyllum ligulaefolium (E.B. Bartram) W.R. Buck and Squamidium nigricans (Hook.) Broth.

\section{ORTHOTRICHACEAE}

Macromitrium carions Müll. Hal., Bull. Herb. Boissier 5: 109. 1897.

Illustration: Sharp et al. (1994) como Macromitrium 
altituberculosum Bartr. ex Grout.

Specimens examined: BRASIL. Goiás: Formoso, sobre rochas, junto a fonte termal na Serra Dourada, 2/IX/1979, D.M. Vital 8535p.p. (SP 147842); idem, Fazenda Murici, sobre galhos de piquizeiro, próximo a lagoa do Murici, 31/VIII/1979, D.M. Vital 8523p.p. (SP 147838); Alvorada do Norte, on bark of tree, 30/V/1978, D.M. Vital 8257p.p. (SP 146953).

Brazilian distribution: GO (Alvorada do Norte; Formoso; Itaberaí; Aporé), MA, MS, MT, PA, RR, SP and TO (Araguatins, Rio Piranha). Associated with Erythrodontium squarrosum (Müll. Hal.) Paris and Platygyriella densa (Hook.) W.R. Buck.

Macromitrium contextum Hampe, Ann. Sci. Nat. Bot. ser. 5, 4: 331. 1865.

Illustration: Sharp et al. (1994).

Specimens examined: BRASIL. Goiás: Itaberaí, sobre tronco vivo de Bombacaceae, 15/XII/1964, D.M. Vital 302 (SP 89248); idem, sobre tronco, 24/I/1973, D.M Vital 2210 (SP 90536); Catalão, on bark of tree in a cerrado vegetation $\left(18^{\circ} 06^{\prime} \mathrm{S}, 47^{\circ} 52^{\prime} \mathrm{W}\right), 31 / \mathrm{V} / 1978$, D.M. Vital 8298 (SP 146986); Formoso, Fazenda Murici, sobre tronco de arueira no cerradão, 30/VIII/1979, D.M. Vital 8505 (SP 147821).

Brazilian distribution: AM, GO (Aporé; Goiânia), MT and SP. Associated with Bryum argenteum Hedw. and Erythrodontium squarrosum (Müll. Hal.) Paris.

Schlotheimia jamesonii (W.-Arn.) Brid., Bryol. Univ. 1: 742.1826.

Orthotrichum jamesonii W.-Arn., Mem. Soc. Linn. Par. 1: 349.1823.

Illustration: Florschütz (1964) and Sharp et al. (1994).

Specimens examined: BRASIL. Goiás: Luziânia, km 50 , along the BR-40, on bark in isolated tree, 17/V/1976, D.M. Vital 6274 (SP 135243); Jataí, on tree trunks, 21/V/1976, D.M. Vital 6361 (SP 135304).

Brazilian distribution: BA, CE, ES, GO (Alto Paraíso de Goiás), MA, MS, PE, PR, RJ, RS, SC, SP and DF. Associated with Meteorium nigrescens (Hedw.) Dozy \& Molk.

\section{PILOTRICHACEAE}

Callicostella merkelii (Hornsch.) A. Jaeger, Ber. Thätigk. St. Gallischen Naturwiss. Ges. 1875/76: 356. 1877. Hookeria merkelii Hornsch., Fl. Bras. 1(2): 62. 1840.

Illustration: Florschütz-de Waard (1986, as Schizomitrium merkelii (Hornsch.) J. Florsch.).

Specimens examined: BRASIL. Goiás: Formoso $\left(13^{\circ} 37^{\prime} \mathrm{S}, 48^{\circ} 45^{\prime} \mathrm{W}\right)$, immersed in stream at Serra
Dourada, 25/XII/1984, D.M. Vital 12669 (SP 207632). Brazilian distribution: AC, MG, PA, RJ, RR, SC and SP.

Cyclodictyon albicans (Hedw.) Kuntze, Revis. Gen. Pl. 2: 835. 1891.

Leskea albicans Hedw., Spec. Musc. Frond.: 218. 1801.

Illustration: Sharp et al. (1994) and Buck (1998).

Specimens examined: BRASIL. Goiás: Itaberaí, Córrego Rico, Serra da Lobeira, solo e bordos de riacho, 10/VII/1970, D.M. Vital 1704 (SP 90083); Cocalzinho, nos bordos do Córrego do Barreiro, próximo do km 48 da BR-70, Fazenda Paraíso, 8/XII/1985, V.R. Estuqui s.n. (SP 208494).

Brazilian distribution: CE, MG, MS, MT, PR, RJ, RS and SP. Associated with Brachythecium ruderale (Brid.) W.R. Buck and Lejeunea phyllobola Nees \& Mont.

\section{POTTIACEAE}

Anoectangium aestivum (Hedw.) Mitt., J. Linn. Soc. Bot. 12: 175. 1869.

Gymnostomum aestivum Hedw., Spec. Musc. Frond.: 32. 1801.

Illustration: Zander (1993).

Specimens examined: BRASIL. Goiás: "am Rande der Altstadt na zeitweise sickerfeuchter Felswand", $400 \mathrm{~m}$ alt., 8/VI/1987, A. Schäfer-Verwimp \& I. Verwimp 8668 (SP 368173).

Brazilian distribution: SP.

Barbula indica (Hook.) Spreng. ex Steud., Nomencl. Bot. 2: 72.1824.

Tortula indica Hook., Musci Exot. 2: 135. 1819.

Illustration: Zander (1993) and Sharp et al. (1994).

Specimens examined: BRASIL. Goiás: Paraíso do Norte, on bent tree trunk, 17/II/1974, D.M. Vital 3013p.p. (SP 90857).

Brazilian distribution: AC, AM, BA, ES, MG, MS, MT, PA, RJ, RS, SP and DF. Associated with Erythrodontium longisetum (Hook.) Paris.

Didymodon australasiae (Hook. \& Grev.) R.H. Zander, Phytologia 41: 21. 1978.

Tortula australasiae Hook. \& Grev., Edinburgh J. Sci. 1: 301.1824.

Illustration: Sharp et al. (1994) and Jiménez et al. (2005).

Specimens examined: BRASIL. Goiás: Formoso $\left(13^{\circ} 37^{\prime} \mathrm{S}, 48^{\circ} 45^{\prime} \mathrm{W}\right)$ on bank along a stream at Serra Dourada, 25/XII/1984, D.M. Vital 12672 (SP 207642).

Brazilian distribution: PR, RS and SP. 
Gymnostomum aeruginosum Sm., Flora Brit. 3: 1163. 1804.

Illustration: Zander (1993) and Sharp et al. (1994). Specimens examined: BRASIL. Goiás: Formoso $\left(13^{\circ} 37^{\prime} \mathrm{S}, 48^{\circ} 45^{\prime} \mathrm{W}\right)$, on soil at base of large rocks, 1/I/1985, D.M. Vital 12753 (SP 207716); idem, on bank along stream near waterfall, at Serra Dourada, 1/I/1985, D.M. Vital 12769 (SP 207732). Tocantins: Dianópolis, ca. $10 \mathrm{~km}$ de Taipas perto do Morro da Cabeça Branca na Serra de Ouro, rocha, 11/II/1987, J.R. Pirani et al. 1958 (SP 230707, SPF, K).

Brazilian distribution: SP (Diadema).

Plaubelia sprengelii (Schwägr.) R.H. Zander, Bull. Buffalo Soc. Nat. Sci. 32: 176. 1993.

Barbula sprengelii Schwaegr., Spec. Musc. Frond. 1: 64. 1823.

Illustration: Zander (1993).

Specimens examined: BRASIL. Goiás: Morrinhos, on soil, in a sparse forest covering rocky, 10/IV/1976, D.M. Vital 6144 (SP 135139); Formoso, Fazenda Riachão, Cachoeira do Rio Icaçu, ca. $12 \mathrm{~km} \mathrm{NE} \mathrm{de}$ Formoso, rocha arenítica, ca. $30 \mathrm{~m}$ da cachoeira, 26/XII/1998, D.M. Vital s.n. (SP 373099).

Brazilian distribution: BA, MT, PE, RJ, RR, RS and Arquipélago Fernando de Noronha. Associated with Fissidens angustifolius Sull.

Pseudosymblepharis schimperiana (Paris) H.A. Crum, Bryologist 55(2): 139. 1952.

Syrrhopodon schimperianus Paris, Index Bryol. 1254. 1898.

Illustration: Zander (1993).

Specimens examined: BRASIL. Goiás: Formosa $\left(25^{\circ} 23^{\prime} \mathrm{S}, 47^{\circ} 28^{\prime} \mathrm{W}\right)$, rochas, 28/XI/1985, D.M. Vital 13456p.p. (SP 208399).

Brazilian distribution: GO (Itaberaí), MG, MS, MT, PR, RJ, RS, SC, SP and DF. Associated with Hyophila involuta (Hook.) A. Jaeger and Tortella humilis (Hedw.) Jenn.

Tortella humilis (Hedw.) Jenn., Mann. Moss. W. Pennsylv. 96: 13. 1913.

Barbula humilis Hedw., Spec. Musc. Frond.: 116. 1801.

Illustration: Zander (1993).

Specimens examined: BRASIL. Goiás: Formoso $\left(13^{\circ} 37^{\prime} \mathrm{S}, 48^{\circ} 48^{\prime} \mathrm{W}\right)$, sobre paredes de Caverna do riacho quente, 25/XII/1984, D.M. Vital 12665 (SP 207628); idem, Fazenda Murici (13 $\left.{ }^{\circ} 13^{\prime} \mathrm{S}, 48^{\circ} 49^{\prime} \mathrm{W}\right)$, on rocky substrate with thin layer of soil, 28/XII/1984, D.M. Vital 12725 (SP 207688); Formosa $\left(25^{\circ} 23^{\prime} \mathrm{S}, 4^{\circ} 28^{\prime} \mathrm{W}\right.$ ), sobre tronco de árvore viva, nos bordos do rio Itiquira,
28/XI/1985, D.M. Vital 13429p.p. (SP 208372); idem, sobre rochas recebendo os respingos da segunda cachoeira do rio Itiquira, 28/XI/1985, D.M. Vital 13456p.p. (SP 208399).

Brazilian distribution: BA, ES, GO (Moçamedes; Goiânia), MG, MS, PR, RJ, RS, SC, SP and DF. Associated with Frullania neesii Lindenb., Hyophila involuta (Hook.) A. Jaeger and Pseudosymblepharis schimperiana (Paris) H. Crum.

Trichostomum tenuirostre (Hook. \& Taylor) Lindb., Öfvers K. VetenskAkad. Förh. 21: 225. 1864. Weissia tenuirostris Hook. \& Taylor, Muscol. Brit., sec. ed.: 83. 1827.

Illustration: Zander (1993).

Specimens examined: BRASIL. Goiás: Flores de Goiás, on gullied ground in a sparse forest, along a stream $\left(14^{\circ} 55^{\prime} \mathrm{S}, 4^{\circ} 06^{\prime} \mathrm{W}\right), 30 / \mathrm{V} / 1978$, D.M. Vital 8267 (SP 146960); Formoso, on base of tree trunk along $\operatorname{road}\left(15^{\circ} 15^{\prime} \mathrm{S}, 4^{\circ} 12^{\prime} \mathrm{W}\right), 30 / \mathrm{V} / 1978$, D.M. Vital 8280 (SP 146972); idem, $\left(13^{\circ} 12^{\prime} \mathrm{S}, 48^{\circ} 46^{\prime} \mathrm{W}\right)$, on soil in cerrado vegetation, 30/XII/1984, D.M. Vital 12747 (SP 207710).

Brazilian distribution: AM, BA, CE, MT, PA, RO, RR, RS and SP.

Weissia controversa Hedw., Spec. Musc. Frond.: 67. 1801.

Illustration: Zander (1993) and Sharp et al. (1994). Specimens examined: BRASIL. Tocantins: Miranorte, na base do tronco vivo, 16/II/1974, D.M. Vital 3003 (SP 90850).

Brazilian distribution: AM, BA, GO (Natividade), MG, PR, RJ, RS, SC and DF. Associated with Fissidens angustifolius Sull.

Weissia jamaicensis (Mitt.) Grout, Moss Flora N. Amer. 1: 157.1938.

Tortula jamaicensis Mitt., J. Linn. Soc. Bot. 12: 147. 1869.

Illustration: Zander (1993) and Sharp et al. (1994).

Specimens examined: BRASIL. Goiás: Formoso (133ㄱ' $\left.\mathrm{S}, 48^{\circ} 48^{\prime} \mathrm{W}\right)$, em um barranco úmido junto a nascente Água Quente, 25/XII/1984, D.M. Vital 12658 (SP 207621).

Brazilian distribution: BA, ES, MG, PR and SP.

\section{RUTENBERGIACEAE}

Pseudocryphaea domingensis (Spreng.) W.R. Buck, Bryologist 83(4): 455.1980 (1981).

Neckera domingensis Spreng., Sys. Veg. 16, 4(1): 185. 1877. 
Illustration: Yano et al. (1987, as P. flagellifera (Brid.) Britt.) and Sharp et al. (1994).

Specimens examined: BRASIL. Goiás: Silvânia, em árvore da mata, 19/X/1956, Macedo \& Smith 4831 (SP 81611).

Brazilian distribution: AC, AL, AM, BA, ES, MG, MS, MT, PA, PE, PR, RJ, RO, RR, RS, SC and SP.

\section{SEMATOPHYLLACEAE}

Meiothecium boryanum (Müll. Hal.) Mitt., J. Linn. Soc. Bot. 12: 469. 1869.

Neckera boryana Müll. Hal., Syn. Musc. Frond. 2: 75. 1850.

Illustration: Sharp et al. (1994), Florschütz-de Waard (1996) and Buck (1998).

Specimens examined: BRASIL. Goiás: Jataí, on a tree trunk, 21/V/1976, D.M. Vital 6356 (SP 135900); Formoso, Fazenda Murici, sobre tronco de Angelin na lagoa, 31/VIII/1979, D.M. Vital 8528 (SP 147837).

Brazilian distribution: AM, PA, RJ and DF. Associated with Octoblepharum albidum Hedw.

Sematophyllum adnatum (Michx.) E. Britton, Bryologist 5(4): 65. 1902.

Leskea adnata Michx., Flora Boreali/Am. 2: 310. 1803.

Illustration: Sharp et al. (1994) and Buck (1998).

Specimens examined: BRASIL. Goiás: Formoso $\left(13^{\circ} 37^{\prime} \mathrm{S}, 48^{\circ} 45^{\prime} \mathrm{W}\right)$, on banks along a stream, 25/XII/1984, D.M. Vital 12688 (SP 207651).

Brazilian distribution: AM, BA, ES, MT, PA, RJ, RS, SP, TO (Jalapão) and DF.

Sematophyllum subpinnatum (Brid.) E. Britton, Bryologist 21(2): 28. 1918 (1919).

Leskea subpinnata Brid., Musc. Recent Suppl. 2: 54. 1812.

Illustration: Sharp et al. (1994) and Buck (1998). Specimens examined: BRASIL. Tocantins: Miracema do Norte, nas pedras da cachoeira São José, 17/II/1974, D.M. Vital 3005 (SP 90852).

Brazilian distribution: AC, AM, AP, BA, CE, ES, GO (Itaberaí; Corumbá de Goiás; Formoso; São João da Aliança; Alto Paraíso de Goiás; Aporé; Rio Quente), MG, MS, MT, PA, PB, PE, PR, RJ, RO, RR, RS, SC, SP, TO (Goiatins; Jalapão) and DF.

\section{SPHAGNACEAE}

Sphagnum rotundatum Müll. Hal. \& Warnst. ex Warnst., Hedwigia 36: 162. 1897.

Illustration: Yano et al. (1985).

Specimens examined: BRASIL. Goiás: Posse, ca.
$7 \mathrm{~km} \mathrm{~S}$ from Posse town, crescendo quase submerso, 8/II/1967, D.M. Vital 1155 (SP 89603).

Brazilian distribution: MG, PR, RJ, SC and SP.

\section{THUIDIACEAE}

Thuidium tomentosum Schimp. ex Besch., Mém. Soc. Sci. Nat. Cherbourg 16: 237. 1872.

Illustration: Sharp et al. (1994) and Buck (1998).

Specimens examined: BRASIL. Goiás: Formoso, ca. $3 \mathrm{~km}$ do Riacho Esmeril, Fazenda Matinha, sobre pedras, 20/X/1967, D.M. Vital 1289 (SP 89779); Paraíso do Norte, base of tree trunk, 17/II/1974, D.M. Vital 3012 (SP 90856); Montividiu do Norte, tronco de Jatobá e xixás, Fazenda Ipê Amarelo, 23/XII/1998, D.M. Vital s.n. (SP 373074).

Brazilian distribution: ES, MS, MT, PE, PR, RJ, RR, RS and SP.

\section{References}

Allen, B. 1999. The genus Philonotis (Bartramiaceae, Musci) in Central America. Haussenechtia Beiheft 9: 19-36.

Buck, W.R. 1998. Pleurocapous mosses of the West Indies. Memoirs of The New York Botanical Garden 82: 1-400.

Buck, W.R. \& Goffinet, B. 2000. Morphology and classification of mosses. Pp.71-123. In: Shaw, A.J. \& Goffinet, B. (eds). Bryophyte Biology. New York, Cambridge University Press.

Churchill, S.P. \& Linares, C.E.L. 1995. Prodromus Bryologiae NovoGranatensis. Introducion a la flora de musgos de Colombia. Biblioteca Jose Jeronimo Triana 12: 19-24.

Crum, H. 1994. Sphagnopsida. Sphagnaceae. North American Flora. ser. 2, 11: 1-180.

Florschütz, P.A. 1964. The mosses of Suriname. Part 1. Leiden, E.J. Brill.

Florschütz-de Waard, J. 1986. Musci. In: A.L. Stoffers \& J.C. Lindeman (eds.). Flora of Suriname. Leiden, E.J. Brill.

Florschütz-de Waard, J. 1996. Sematophyllaceae. Pp. 384-438. In: A.R.A. Görts-van Rijn (ed.). Flora of the Guianas ser. C 1.

Frahm, J.-P. 1991. Dicranaceae: Campylopoidioideae, Paraleucobryoideae. Flora Neotropica, monograph 54: 1-238.

Gradstein, S.R. \& Costa, D.P. 2003. The Hepaticae and Anthocerothae of Brazil. Memoirs of The New York Botanical Garden 87: 1-318.

Gradstein, S.R.; Schäfer-Verwimp, A. \& Costa, D.P. 2005. The liverworts (Marchantiophyta) of the state of Goiás: Brazil. Revista de Biologia Neotropical 2: 75-108.

Jiménez, J.A.; Ros, R.M.; Cano, M.J. \& Guerra, J. 2005. A new evaluation of the genus Trichostomopsis (Pottiaceae, Bryophyta). Botanical Journal of the Linnean Society 147: 117-127.

Ochi, H. 1980. A revision of the Neotropical Bryoideae, Musci (First Part). The Journal of the Facculty of Education Tottori University, nat. Sci. 29: 49-145.

Ochi, H. 1981. A revision of the Neotropical Bryoideae, Musci (Second Part). The Journal of the Facculty of Education Tottori University, nat. Sci. 30: 21-55.

Peralta, D.F. \& Vital, D.M. 2006. Archidiaceae (Archidiales, Bryophyta) do Brasil. Boletim do Instituto de Botânica 18: 17-32.

Peralta, D.F. \& Yano, O. 2006. Novas ocorrências de musgos (Bryophyta) para o Estado de São Paulo, Brasil. Revista Brasileira de Botânica 29: 49-65. 
Sehnem, A. 1970. Musgos Sul-brasileiros II. Pesquisas, sér. Bot. 28: 1-117.

Sharp, A.J.; Crum, H. \& Eckel, P.M. 1994. The moss flora of Mexico. Memoirs of The New York Botanical Garden 69: $1-1113$.

Snider, J.A. 1975. A revision of the genus Archidium (Musci). The Journal of the Hattori Botanical Laboratory 39: 105-201.

Yano, O. 1979. Contribuição ao inventário dos Musci Brasileiros: Helicophyllaceae. Rickia 8: 7-16.

Yano, O. 1981. Aytoniaceae (Marchantiales, Hepaticopsida) no Brasil. Revista Brasileira de Botânica 4: 89-94.

Yano, O. 1989. An additional checklist of Brazilian bryophytes. The Journal of the Hattori Botanical Laboratory 66: 371-434.

Yano, O. 1995. A new additional annotated checklist of Brazilian bryophytes. The Journal of the Hattori Botanical Laboratory 78: 137-182.

Yano, O. 2006. Novas adições ao catálogo de briófitas brasileiras. Boletim do Instituto de Botânica 17:1-142.
Yano, O. \& Bordin, J. 2006. Novas ocorrências de briófitas para o Rio Grande do Sul, Brasil. Boletim do Instituto de Botânica 18: $111-122$.

Yano, O. \& Costa, D.P. 2000. Flora dos Estados de Goiás e Tocantins: Criptógamos. v.5. Briófitas. Goiânia. Cegraf/UFG.

Yano, O. \& Oliveira-e-Silva, M.I.M.N. 1997. Criptógamos do Parque Estadual das Fontes do Ipiranga, São Paulo, SP. Briófitas 2: Fissidentaceae (Bryales). Hoehnea 24: 107-114.

Yano, O. \& Peralta, D.F. 2007. Flora dos Estados de Goiás e Tocantins: Criptógamos: Musgos (Bryophyta). J.A. Rizzo (coord.).

Yano, O.; Marinho, M.G.V. \& Mariz, G. 1987. Novas ocorrências de briófitas no nordeste brasileiro. Rickia 14: 73-87.

Yano, O.; Pirani, J.R. \& Santos, D.P. 1985. O gênero Sphagnum (Bryopsida) nas regiões sul e sudeste do Brasil. Revista Brasileira de Botânica 8: 55-80.

Zander, R.H. 1993. Genera of the Pottiaceae: mosses of harsh environments. Bulletin of the Buffalo Society of Natural Sciences 32: 1-378. 